\title{
Toward Atomic-Scale Tomography: The ATOM Project
}

\author{
T. F. Kelly, M. K. Miller ${ }^{\mathrm{ii}}$, K. Rajan ${ }^{\mathrm{iii}}$, S. P. Ringer ${ }^{\mathrm{iv}}$, A.Y. Borisevich ${ }^{\mathrm{ii}}$, N. Dellby ${ }^{\mathrm{v}}$, \\ and O.L. Krivanek ${ }^{\mathrm{v}}$
}

${ }^{\text {i}}$ Cameca Instruments, Inc., 5500 Nobel Drive, Madison, WI 53726

ii Materials Science and Technology Division, Oak Ridge National Laboratory, Oak Ridge, TN 37831

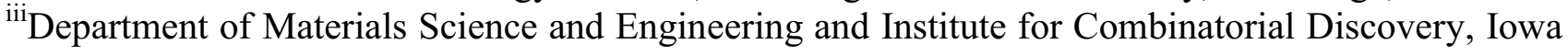
State University, 2220 Hoover Hall, Iowa State University, Ames, IA 50011

${ }^{\text {iv }}$ Australian Centre for Microscopy \& Microanalysis, University of Sydney, NSW 2006, Australia

vNion Company, 1102 8th St., Kirkland, WA 98033

It is instructive to consider what constitutes the ultimate microscope. It must be able to image accurately the position and identity of every atom (or isotope) in a material so that a complete microstructural understanding is realized. But can such a microscope be built? Our analysis shows that neither scanning transmission electron microscopy (STEM) nor atom probe tomography (APT), which work fundamentally via 3D projection and 3D tomography, respectively, will achieve this independently. We envision a new branch of microscopy whereby these complementary techniques are combined in a new concept, ,atomic-resolution tomographic microscopye - the Atomscope. Our vision is for a new instrument that will deliver 3D atomic resolution chemical imaging not only of pristine crystals and „standard"e specimens, but for practical technological materials. We foresee identifying the exact 3D position and chemical identity of every atom and defect throughout large volumes, generating high-resolution datasets containing billions of atoms.

If this ultimate microscope can be built, what impact will it have on scientific discovery? In the fields of quantum computing, nanoelectronics, advanced metallurgy, etc., single atoms are known to alter the behavior of todayes devices. Since engineering structures are being fabricated at the single atom level, atomic-scale tomography is needed already for both science and technology.

Our mission is to build a working Atomscope with three specific aims:

1. Perfectly reconstruct APT images by:

a. Fusing the data from electron microscopy and atom probe tomography.

b. Developing radically new reconstruction algorithms.

2. Detect all atoms in atom probe tomography (achieve near $100 \%$ efficient data collection).

3. Build a fully functional Atomscope by:

a. Realizing the synergies of the combination of TEM/APT combination.

b. Developing mining methods for the fused data.

The single most significant limitation of atom probe tomography today is data reconstruction. In principle, if the shape of the actual specimen is fully known, then the projection can be performed correctly. It appears that STEM images in projection will have enough spatial resolution. Since a 3D surface is desired, we will design the stage to be capable of $360^{\circ}$ rotation of the specimen about its major axis. Extensive analytical methods must be coupled with STEM images of the specimen such that the information is used to produce superior reconstruction results. The image processes in local electrode atom probe (LEAP) and STEM are currently open loop. However, the combination of these two complementary techniques creates a closed-loop imaging process, and this generates information that neither instrument is capable of producing when operated independently.

In the LEAP, all atoms field evaporate but only $\sim 40-60 \%$ of the atoms are detected. We aim to pursue approaches for improving the detection efficiency for APT. A near-term potential solution seeks to improve microchannel plates and may get us to greater than $80 \%$ detection efficiency. With 
superconducting detectors [1], there appears to be the opportunity to configure a detector [2] which has (a) full areal coverage (100\% detection efficiency), (b) a physical process that is fundamentally fast enough to allow sub-100 ps timing, and (c) curiously, a signal that may be proportional to the kinetic energy of the incoming ion. It is not yet clear which mechanism, if any, will work for APT detectors. A serious challenge will be to make the impact position detectable in both $\mathrm{X}$ and $\mathrm{Y}$.

The environmental constraints on STEM systems are far greater than those on atom probes. After careful consideration, we are convinced that the surest route is to start with a working STEM and add a LEAP. Furthermore, a state-of-the-art commercial STEM, the Nion UltraSTEM ${ }^{\text {TM }} 200$, is modular in design, which makes adaptation of a LEAP sufficiently straightforward that there does not appear to be any reason to begin a design from scratch. Given these mutual constraints and the design of the Nion STEM, we are primarily concerned about four aspects: 1. specimen shape must be a needle to enable LEAP operation, 2. achieving vibration-free, cryogenic operation, 3 . achieving "drift-free" specimen articulation, and 4. operation under ultrahigh vacuum conditions.[3]

[1] K. D. Irwin, Scientific American, (November 2006) 86-94.

[2] T.F. Kelly, Microsc. Microanal. 17 (2011) 1.

[3] Support for this work comes from ONR-MURI Award: NN0014-06-1-1176 and NSF awards: PHY CDI- 09-41576, CMMI-ARI- 09-389018 and CCF-AF- 09-17202 (KR) and the Australian Research Council (SPR). Research at ORNL's Shared Research Equipment (SHaRE) User Facility is sponsored by the Office of Basic Energy Sciences, U.S. Department of Energy (MKM, AYB).

a)

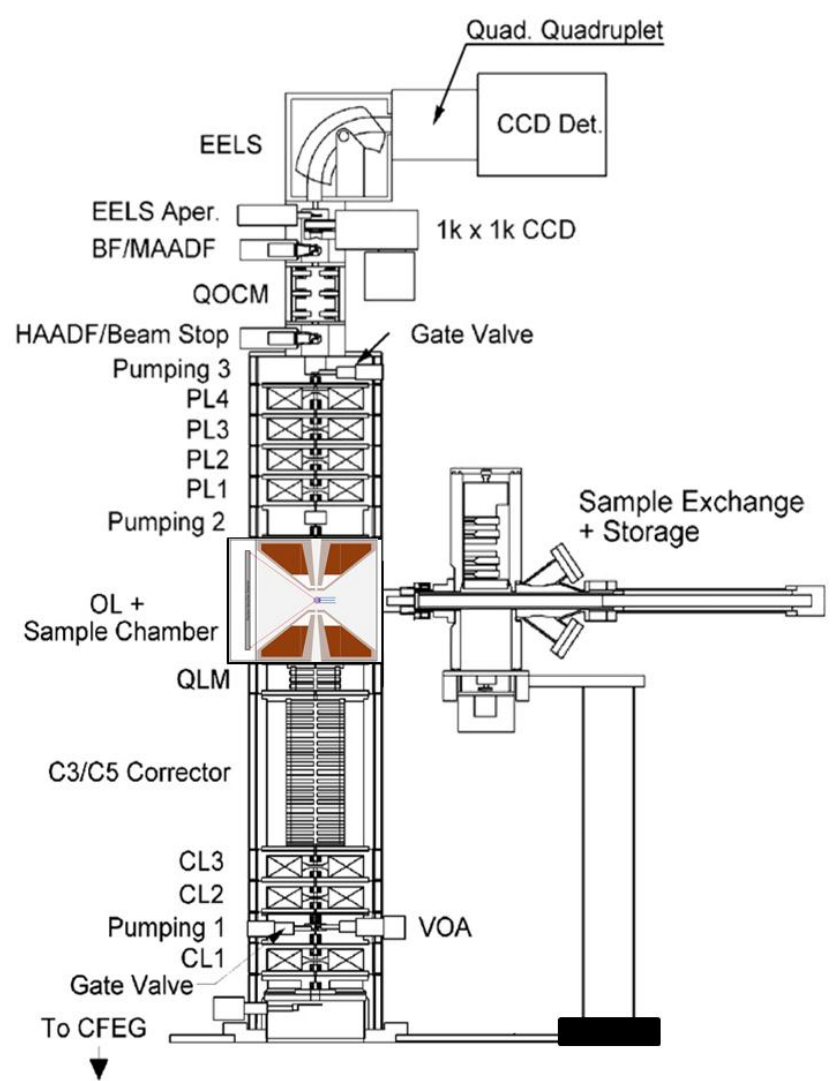

b)

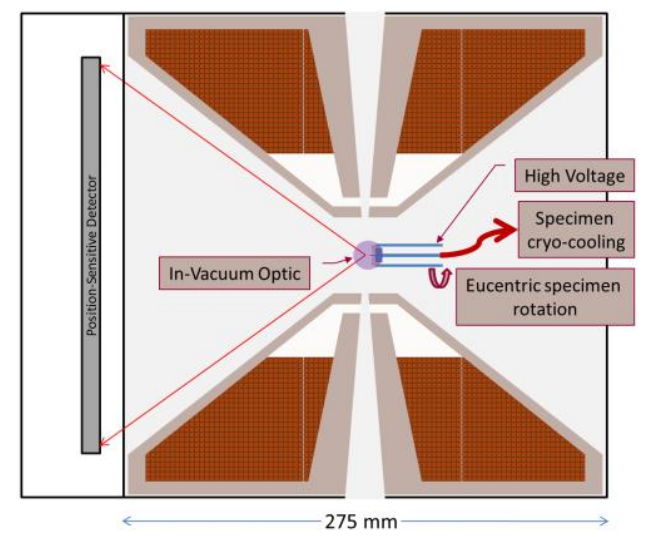

c)

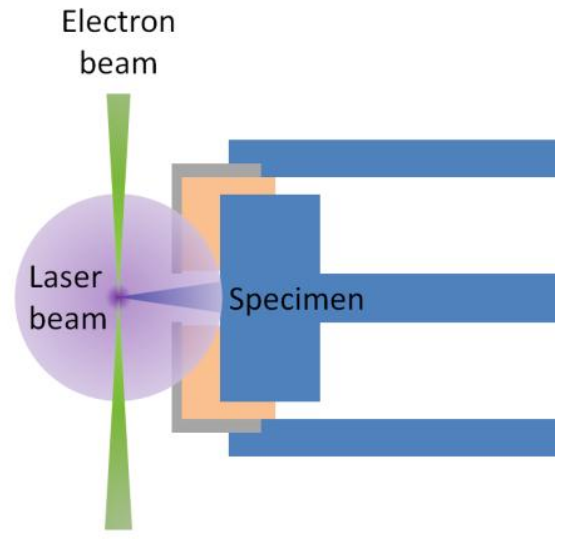

FIG. 1. a) Schematic of Nion UltraSTEM 200 column with LEAP added to objective lens/sample chamber. b) The objective lens/LEAP module. c) The LEAP specimen in the object plane of the STEM objective lens. 